\title{
Impulsive Containment Control in Nonlinear Multiagent Systems with Time-Delay
}

\author{
Wenshan Hu, Zhenhua Wang, Zhi-Wei Liu, and Hong Zhou \\ Department of Automation, Wuhan University, Wuhan 430072, China \\ Correspondence should be addressed to Zhi-Wei Liu; liuzw@whu.edu.cn
}

Received 12 June 2014; Accepted 8 August 2014

Academic Editor: Housheng Su

Copyright (C) 2015 Wenshan Hu et al. This is an open access article distributed under the Creative Commons Attribution License, which permits unrestricted use, distribution, and reproduction in any medium, provided the original work is properly cited.

\begin{abstract}
The containment control problems of nonlinear multiagent systems with time-delay via impulsive algorithms under both fixed and switching topologies are studied. By using the Lyapunov methods, several conditions are derived to achieve the containment control. It is shown that the states of the followers can converge into the convex hull spanned by the states of the leaders if every leader has directed paths to all the followers and the impulsive period is short enough. Finally, some simulations are conducted to verify the effectiveness of the proposed algorithms.
\end{abstract}

\section{Introduction}

Recently, the distribution cooperative control of the multiagent systems has attracted numerous researches due to the extensive applications in the scientific, engineering, biological aspects, and so forth [1-5]. The very essence of the distribution cooperative control lies in that none of the agents know the states of all the other agents exactly and every agent updates its own states based on the state information from its neighbors. Consensus problems, as a fundamental branch of cooperative control, have been investigated in many literatures with plenty of useful results obtained [6, 7]. In earlier times, the researches mainly focused on the consensus cases where there exists no more than one leader [8-11]. Zhu and Cheng in [8] considered the consensus of multiagent systems where there exists one leader. Su et al. in $[9,10]$ mainly investigated the leader-following consensus of linear multiagent systems with one leader and input saturation. Lu et al. [11] studied the finite-time distributed tracking control for multiagent systems with an active leader.

The investigations on consensus problems of multiple leaders [12-19], called containment control, are also significant because they are related with many practical applications, such as there are some agents equipped with the detective sensors, while the other less capable agents are driven into the convex hull spanning by the former agents such that all the agents can navigate their trip without any potential danger.

The proposed algorithms in $[13,14]$ are applied to multiagent systems with linear dynamics. These critical defects limit the validity of the proposed models because the nonlinear systems are ubiquitous in the real world [20-22]. In this paper, we deal with the containment control of the nonlinear multiagent systems. Compared with the continuous control algorithms [12-19], the impulsive algorithms have remarkable superiority as the impulsive controllers are usually simple and practical [23-26]. In many cases, to achieve the consensus, the continuous regulation and control are not necessary or impossible. Nevertheless, the impulsive algorithms just need to regulate and alter the states of the systems at the impulsive instants. Also, it can be easily seen that impulsive control is effective and efficient in the control of the multiagent systems to achieve consensus. In many real applications, due to the limited switching speed and the transmission of the signals, the next states of the dynamic systems may be concerned not only with the states at this time but also with states of the time before. It is practical and necessary to analyse the systems with time-delay. If the time-delay is not taken into consideration in the system models, inappropriate or even inaccurate results may be obtained. There are some works 
$[27,28]$ that deal with the consensus or synchronization problem with time-delays.

Motivated by the aforementioned discussions, we investigate the achievement of the containment control for the nonlinear multiagent systems with time-delay under both fixed and switching topology in this paper. The proposed impulsive algorithm can achieve the containment control and some sufficient conditions are obtained, which show that, for specific dynamic systems, the containment control can be achieved if the control period of the impulsive algorithms and the feedback gain of the controller satisfy the given conditions. Finally, some numerical simulations are presented to verify the validity of the theoretical analysis.

The outline of this paper is organized as follows. In Section 2, we first give some preliminaries which are essential for the analyses of the multiagent systems. Then, the containment control of the nonlinear dynamic systems with internal time-delays under both fixed and switching topology via impulsive algorithms is considered. In Section 3, some simulations are presented to illustrate the theoretical results. Finally, some conclusions of this paper are drawn in Section 4

The notions and symbols used in the paper are given as follows. $\mathbb{R}$ denotes the set of real numbers. Let $I_{N}$ be the $N \times N$ identity matrix (or just $I$ if no confusion), $\mathbf{1}_{N}=[1,1, \ldots, 1]^{T}$. $\mathbf{0}_{N \times M}$ is the $N \times M$ matrix with all entries zero. $\|\cdot\|$ denotes the Euclidean norm. $\lambda_{\text {max }}(A), \lambda_{\text {min }}(A)$ denote the maximum and minimum eigenvalue of the square matrix $A$, respectively.

\section{Impulsive Containment Control for Nonlinear Systems with Internal Delay}

First, we summarize some definitions and results from graph theory that will be used in the paper.

Let $\mathscr{G}=(\mathscr{V}, \mathscr{E}, \mathscr{A})$ be a weighted graph consisting of the set of nodes $\mathscr{V}=\{1,2, \ldots, n\}$, the set of edges $\mathscr{E} \subset$ $\mathscr{V} \times \mathscr{V}$, and a weighted adjacency matrix $\mathscr{A}=\left[a_{i j}\right], i$, $j=1,2, \ldots, n$. If $(j, i) \in \mathscr{E}$, then $a_{i j}>0$ and $a_{j i}>0$; otherwise, $a_{i j}=a_{j i}=0$ and the diagonal entries of $\mathscr{A}$ are zero; that is, $a_{i i}=0$. A path from node $i$ to node $j$ is a sequence of edges $\left(i, p_{1}\right),\left(p_{1}, p_{2}\right), \ldots,\left(p_{k}, j\right)$, where $\left(i, p_{1}\right),\left(p_{1}, p_{2}\right), \ldots,\left(p_{k}, j\right) \in \mathscr{E} . \mathcal{N}_{i}$ denotes the set of the neighbors of node $i$, where $\mathcal{N}_{i}=\{j \in \mathscr{V}:(j, i) \in \mathscr{E}\}$. The Laplacian matrix of graph $\mathscr{G}$ is denoted by $L=\left[l_{i j}\right] \in \mathbb{R}^{n \times n}$, $l_{i i}=\sum_{j=1}^{n} a_{i j}$ and $l_{i j}=-a_{i j}$ for $i \neq j$.

\subsection{Impulsive Containment Control for Nonlinear Dynamic} Systems of Fixed Topology with Internal Delay. Consider a nonlinear dynamic multiagent system with $(M+N)$ agents. Assume that there are $M$ leaders and $N$ followers. An agent is a leader if the agent does not receive any information from others. Otherwise, it is a follower. The set of leaders is denoted by $\mathscr{M}=\{1,2, \ldots, M\}$ and the set of followers is denoted by $\mathcal{N}=\{M+1, \ldots, M+N\}$. Let $\mathscr{V}=\mathscr{M} \cup \mathcal{N}=\{1,2, \ldots, M+N\}$ be the set of all the agents. The communications among the $(M+N)$ agents are represented by a directed graph $\mathscr{G}$.
The dynamics of the nonlinear multiagent system with internal time-delay are given as follows:

$$
\begin{aligned}
& \dot{x}_{i}(t)=g\left(t, x_{i}(t), x_{i}(t-d)\right)+u_{i}(t), \quad i \in \mathcal{N}, \\
& \dot{x}_{i}(t)=g\left(t, x_{i}(t), x_{i}(t-d)\right), \quad i \in \mathscr{M},
\end{aligned}
$$

where $d$ is the internal delay and $x_{i}(t) \in \mathbb{R}^{n}$ and $u_{i}(t) \in \mathbb{R}^{n}$ are the state and control input of the $i$ th agent, respectively. $g(\cdot, \cdot, \cdot): \mathbb{R} \times \mathbb{R}^{n} \times \mathbb{R}^{n} \rightarrow \mathbb{R}^{n}$ is the nonlinear dynamic of the $i$ th agent.

Assumption 1. The nonlinear function $g\left(t, x_{i}(t), x_{i}(t-d)\right)$ in system (1) satisfies the convex Lipschitz condition. That is, there exist two positive numbers $K$ and $l$, such that, for all $x_{i}, y_{i}, i=M+1, \ldots, M+N$,

$$
\begin{aligned}
& \left\|g\left(t, x_{i}, y_{i}\right)-\sum_{j=1}^{M} a_{j} g\left(t, x_{j}, y_{j}\right)\right\| \\
& \quad \leqslant K\left\|x_{i}-\sum_{j=1}^{M} a_{j} x_{j}\right\|+l\left\|y_{i}-\sum_{j=1}^{M} a_{j} y_{j}\right\|,
\end{aligned}
$$

where $a_{j} \geqslant 0, j=1, \ldots, M$ and $\sum_{j=1}^{M} a_{j}=1$.

The initial states of the system (1) are

$$
x_{i}(t)=o_{i}(t), \quad-d \leqslant t \leqslant 0, i \in \mathscr{V},
$$

where $o_{i}(t) \in C\left([-d, 0], \mathbb{R}^{n}\right)$ and $C\left([-d, 0], \mathbb{R}^{n}\right)$ is the $n$ dimensional vector space of continuous functions. In this paper, we consider the case of $n=1$ and all the results will hold for $x_{i}(t), u_{i}(t) \in \mathbb{R}^{n}(n>1)$ by using the property of Kronecker product.

From the definitions of the leader and the follower, the Laplacian matrix of the graph $\mathscr{G}$ can be partitioned as

$$
L=\left[\begin{array}{cc}
L_{11} & L_{12} \\
0_{M \times N} & 0_{M \times M}
\end{array}\right],
$$

where $L_{11}$ is a $N \times N$ matrix which denotes the communications among the followers. $L_{12}$ is a $N \times M$ matrix which denotes the communications among the followers and leaders.

Assumption 2. In the graph $\mathscr{G}$, the communications among the followers are undirected and, for each follower, there exists at least one leader that has a directed path to that follower.

From the property of the Laplacian matrix, the following lemma can be obtained.

Lemma 3 (see [17]). Let Assumption 1 hold. Then $L_{11}$ is symmetric positive definite and all the entries of $-\left(L_{11}^{-1} L_{12}\right)$ are nonnegative and $-\left(L_{11}^{-1} L_{12}\right) \mathbf{1}_{M}=\mathbf{1}_{N}$.

Definition 4 (see [29]). A subset $C$ of $\mathbb{R}^{N}$ is said to be convex if $(1-\lambda) x+\lambda y \in C$ whenever $x \in C, y \in C$, and $0<\lambda<1$. 
The convex hull of a finite set of points $x_{1}, x_{2}, \ldots, x_{q} \in \mathbb{R}^{N}(q$ is a positive integer) is the minimal convex set containing all points in $\left\{x_{1}, x_{2}, \ldots, x_{q}\right\}$. We use $\operatorname{co}\left\{x_{1}, x_{2}, \ldots, x_{q}\right\}$ to denote it; that is,

$$
\operatorname{co}\left\{x_{1}, x_{2}, \ldots, x_{q}\right\}=\left\{\sum_{j=1}^{q} a_{j} x_{j} \mid a_{j} \in \mathbb{R}, a_{j} \geqslant 0, \sum_{j=1}^{q} a_{j}=1\right\} .
$$

Definition 5. The containment control is achieved in system (1) if, for any initial conditions, $x_{i}(t) \in \operatorname{co}\left\{x_{1}(t), \ldots, x_{M}(t)\right\}$ as $t \rightarrow+\infty$, for all $i \in \mathcal{N}$. That is, each state of the followers will converge into the convex hull formed by the states of the leaders as $t \rightarrow+\infty$.

To achieve the containment control for the system (1), we propose the following impulsive algorithm:

$$
u_{i}(t)=\sum_{k=1}^{\infty}\left[(-\alpha) \sum_{j=1}^{M+N} a_{i j}\left(x_{i}(t)-x_{j}(t)\right)\right] \delta\left(t-t_{k}\right)
$$

$$
i \in \mathcal{N}
$$

where $\alpha>0$ is the feedback gain to be determined and $\mathscr{A}=$ $\left[a_{i j}\right] \in \mathbb{R}^{n \times n}$ is the adjacency matrix associated with the graph $\mathscr{G}$. The impulsive instants are $t_{k}, k=1,2, \ldots, \lim _{k \rightarrow+\infty} t_{k}=$ $+\infty$. That is, $t_{1}<t_{2}<\cdots<t_{k}<\cdots$ is the impulsive instant sequence, where $t_{1}>t_{0} ; t_{0}=0$ is the initial time. $\delta(\cdot)$ is the Dirac impulsive function.

Using the impulsive algorithm (6) in the system (1), we can obtain

$$
\begin{aligned}
\dot{x}_{i}(t) & =g\left(t, x_{i}(t), x_{i}(t-d)\right), \quad t \neq t_{k}, \\
\Delta x_{i}\left(t_{k}\right) & =(-\alpha) \sum_{j=1}^{N+M} a_{i j}\left(x_{i}\left(t_{k}\right)-x_{j}\left(t_{k}\right)\right), \\
& i \in \mathcal{N} \\
\dot{x}_{i}(t) & =g\left(t, x_{i}(t), x_{i}(t-d)\right), \quad t>0, i \in \mathscr{M},
\end{aligned}
$$

where $\Delta x_{i}\left(t_{k}\right)=x_{i}\left(t_{k}^{+}\right)-x_{i}\left(t_{k}\right)$ and $x_{i}\left(t_{k}^{+}\right)=\lim _{t \rightarrow t_{k}^{+}} x_{i}(t)$. Assume that $x_{i}(t)$ is left-hand continuous at $t=t_{k}, k=$ $1,2, \ldots$; that is, $x_{i}\left(t_{k}^{-}\right)=x_{i}\left(t_{k}\right)$, where $x_{i}\left(t_{k}^{-}\right)=\lim _{t \rightarrow t_{k}^{-}} x_{i}(t)$, $i \in \mathcal{N}$. For simplification, we assume that $x_{i}(t), i \in \mathscr{V}$, is continuous at the initial time $t_{0}=0$.

Assumption 6. The internal delay must be less than the impulsive period. That is, $0 \leqslant d \leqslant \tau_{0}$, where $t_{k+1}-t_{k}=\tau_{0}$, $k=1,2 \ldots$, is the impulsive period.
Let

$$
\begin{aligned}
& x_{F}(t)= {\left[x_{M+1}(t), \ldots, x_{M+N}(t)\right]^{T}, } \\
& x_{L}(t)=\left[x_{1}(t), \ldots, x_{M}(t)\right]^{T}, \\
& G_{F}(t)=\left[g\left(t, x_{M+1}(t), x_{M+1}(t-d)\right), \ldots,\right. \\
&\left.g\left(t, x_{M+N}(t), x_{M+N}(t-d)\right)\right]^{T}, \\
& G_{L}(t)=\left[g\left(t, x_{1}(t), x_{1}(t-d)\right), \ldots,\right. \\
&\left.g\left(t, x_{M}(t), x_{M}(t-d)\right)\right]^{T} .
\end{aligned}
$$

According to equality (4), the system (7) can be rewritten as

$$
\begin{aligned}
\dot{x}_{F}(t) & =G_{F}(t), \quad t \neq t_{k} \\
\Delta x_{F}\left(t_{k}\right) & =-\alpha L_{11}\left[x_{F}\left(t_{k}\right)+L_{11}^{-1} L_{12} x_{L}\left(t_{k}\right)\right], \\
\dot{x}_{L}(t) & =G_{L}(t), \quad t>0 .
\end{aligned}
$$

Let $\omega(t)=x_{F}(t)+\left(L_{11}^{-1} L_{12}\right) x_{L}(t), t \neq t_{k}$, and $\Delta \omega\left(t_{k}\right)=$ $\Delta x_{F}\left(t_{k}\right)+\left(L_{11}^{-1} L_{12}\right) \Delta x_{L}\left(t_{k}\right)$. Because $\Delta x_{L}\left(t_{k}\right)=0$ and from equality (9), the following equations can be obtained:

$$
\begin{aligned}
\dot{\omega}(t) & =G_{F}(t)+\left(L_{11}^{-1} L_{12}\right) G_{L}(t), \quad t \neq t_{k}, \\
\Delta \omega\left(t_{k}\right) & =-\alpha L_{11} \omega\left(t_{k}\right) .
\end{aligned}
$$

Remark 7. From Lemma 3, every entry in $-\left(L_{11}^{-1} L_{12}\right)$ is nonnegative and $-\left(L_{11}^{-1} L_{12}\right) \mathbf{1}_{M}=\mathbf{1}_{N}$. We can get that each entry of $\left(-L_{11}^{-1} L_{12}\right) x_{L}(t)$ is the convex hull formed by the states of the leaders. So, the containment control is achieved if the system (10) is stable.

Theorem 8. Suppose that Assumptions 1, 2, and 6 are satisfied. The system (1) using impulsive algorithm (6) achieves containment control, that is, $x_{F}(t) \rightarrow\left(-L_{11}^{-1} L_{12}\right) x_{L}(t)$, as $t \rightarrow+\infty$, if the following conditions are satisfied:

$$
\begin{gathered}
\frac{\lambda_{\text {max }}\left(L_{11}\right)-\lambda_{\text {min }}\left(L_{11}\right)}{\lambda_{\text {max }}\left(L_{11}\right)+\lambda_{\text {min }}\left(L_{11}\right)} \leqslant q<1, \\
\frac{1-q}{\lambda_{\text {min }}\left(L_{11}\right)} \leqslant \alpha \leqslant \frac{1+q}{\lambda_{\text {max }}\left(L_{11}\right)}, \\
\tau_{0}<\frac{-\ln (q)}{2(K+l)},
\end{gathered}
$$

where $q$ is an auxiliary constant.

Proof. Construct a Lyapunov function $V(t)=\omega^{T}(t) \omega(t)$. Then $V(t)$ is piecewise continuous and positive definite. When $t \neq t_{k}$, we can get $\dot{V}(t)=2 \omega^{T}(t) \dot{\omega}(t)$. Let $\omega(t)=$ $\left[\omega_{1}(t), \ldots, \omega_{N}(t)\right]^{T}$ and $\bar{\omega}(t)=\left[\left|\omega_{1}(t)\right|, \ldots,\left|\omega_{N}(t)\right|\right]^{T}$; then we can get $V(t)=\bar{\omega}^{T}(t) \bar{\omega}(t)$. From Assumption 1 and (10),

$$
\dot{V}(t) \leqslant 2 \bar{\omega}^{T}(t)(K \bar{\omega}(t)+l \bar{\omega}(t-d))
$$


can be obtained. That is,

$$
\dot{V}(t) \leqslant 2 K \bar{\omega}^{T}(t) \bar{\omega}(t)+2 l \bar{\omega}^{T}(t) \bar{\omega}(t-d) .
$$

It is obvious that

$$
2 \bar{\omega}^{T}(t) \bar{\omega}(t-d) \leqslant \bar{\omega}^{T}(t) \bar{\omega}(t)+\bar{\omega}^{T}(t-d) \bar{\omega}(t-d) .
$$

Combining inequalities (15) and (16) and the definition of $V(t)$, we can arrive at the following inequality:

$$
\dot{V}(t) \leqslant(2 K+l) V(t)+l V(t-d), \quad t \neq t_{k} .
$$

From the definition of $V(t)$, we can obtain $V\left(t_{k}^{+}\right)=$ $\omega^{T}\left(t_{k}^{+}\right) \omega\left(t_{k}^{+}\right)$. From system $(10)$, it is easy to see that $\omega\left(t_{k}^{+}\right)=$ $\left(I-\alpha L_{11}\right) \omega\left(t_{k}\right)$. Thus,

$$
\begin{aligned}
V\left(t_{k}^{+}\right) & =\omega^{T}\left(t_{k}\right)\left(I-\alpha L_{11}\right)^{T}\left(I-\alpha L_{11}\right) \omega\left(t_{k}\right), \\
& \leqslant \lambda_{\max }^{2}\left(I-\alpha L_{11}\right) \omega^{T}\left(t_{k}\right) \omega\left(t_{k}\right) .
\end{aligned}
$$

That is,

$$
V\left(t_{k}^{+}\right) \leq \lambda_{\max }^{2}\left(I-\alpha L_{11}\right) V\left(t_{k}\right) .
$$

To prove that the Lyapunov function $V(t)$ is convergent, we construct an auxiliary variable $\xi$ in the following form:

$$
\begin{aligned}
\dot{\xi}(t) & =(2 K+l) \xi(t)+l \xi(t-d)+\mu, \quad t \neq t_{k} \\
\xi\left(t_{k}^{+}\right) & =\lambda_{\max }^{2}\left(I-\alpha L_{11}\right) \xi\left(t_{k}\right),
\end{aligned}
$$

where the initial states are $\xi(t)=\left\|o_{\omega}(t)\right\|^{2},-d \leqslant t \leqslant 0$, and $\mu>0$, where $o_{\omega}(t)=o_{F}(t)+\left(L_{11}^{-1} L_{12}\right) o_{L}(t), o_{F}(t)=$ $\left[o_{M+1}(t), \ldots, o_{M+N}(t)\right]^{T}$, and $o_{L}(t)=\left[o_{1}(t), \ldots, o_{M}(t)\right]^{T}$.

Let

$$
\begin{aligned}
& \dot{\eta}(t)=(2 K+l) \eta(t), \quad t \neq t_{k}, \\
& \eta\left(t_{k}^{+}\right)=\lambda_{\text {max }}^{2}\left(I-\alpha L_{11}\right) \eta\left(t_{k}\right) .
\end{aligned}
$$

From inequalities (17), (19), and (20), it is obvious that, for all $t>0,0 \leqslant V(t) \leqslant \xi(t)$. And the solution of (20) satisfies the following integral equation:

$$
\xi(t)=\phi(t, 0) \xi(0)+\int_{0}^{t} \phi(t, s)[l \xi(s-d)+\mu] d s,
$$

where $\phi(t, s),(t, s \geqslant 0)$, is the state transition matrix of the system (21).

When $t_{k}<t<t_{k+1}$ and $t_{k-1}<s<t_{k}, k=1,2, \ldots$, we can arrive at

$$
\phi(t, s) \leqslant e^{(2 K+l)(t-s)} \lambda_{\max }^{2}\left(I-\alpha L_{11}\right) .
$$

From inequalities (11) and (12), we can obtain

$$
\left|\lambda_{\max }\left(I-\alpha L_{11}\right)\right| \leqslant q<1 .
$$

Let $a=-(2 K+l)-\gamma / 2$ and $2 \ln (q) / \tau_{0}=\gamma$. From condition (13), it is obvious that $\gamma<-4(K+l)$ and $a>0$. Accordingly, inequality (23) can be rewritten:

$$
\begin{aligned}
\phi(t, s) & \leqslant e^{(-a-\gamma / 2)(t-s)} \lambda_{\max }^{2}\left(I-\alpha L_{11}\right) \\
& <e^{-a(t-s)} \cdot e^{-(\gamma / 2) \cdot 2 \tau_{0}} \lambda_{\max }^{2}\left(I-\alpha L_{11}\right) \leqslant e^{-a(t-s)} .
\end{aligned}
$$

Thus, from equality (22), we can obtain

$$
\xi(t)<e^{-a t}\left\|\underset{\omega}{\sup (-d \leqslant t \leqslant 0)} o_{\omega}(t)\right\|^{2}+\int_{0}^{t} e^{-a(t-s)}[l \xi(s-d)+\mu] d s
$$

for $t \geqslant 0$.

Let $\varepsilon(\theta)=\theta-a+l e^{\theta d}$. Then, $\varepsilon(\theta)=0$ has a unique solution $\theta^{*}>0$ because $\varepsilon(\theta)$ is a strictly monotonic function $\dot{\varepsilon}(\theta)=$ $1+l d e^{\theta d}>0$ and $\varepsilon(0)=-a+l<0, \varepsilon(+\infty)>0$. According to $a-l>0$ and $-\theta^{*} t>0$ when $-d \leqslant t \leqslant 0$, we can get

$$
\xi(t)<e^{-\theta^{*} t} \underset{\substack{\sup (-d \leqslant t \leqslant 0) \\ \operatorname{soc}}}{\|}(t) \|^{2}+\frac{\mu}{a-l},
$$

where $-d \leqslant t \leqslant 0$. And we can prove that inequality (27) is true for $t \geqslant 0$ in the following.

Suppose that inequality (27) is not held; there must exist a $t_{c}$ such that





However, from (26) and (29), we can obtain

$$
\begin{aligned}
& \xi\left(t_{c}\right)<e^{-a t_{c}}\left(\underset{\substack{\sup (-d \leqslant t \leqslant 0) \\
\operatorname{oos}}}{\|}(t) \|^{2}+\frac{\mu}{a-l}\right) \\
& +\int_{0}^{t_{c}} e^{-a\left(t_{c}-s\right)}[l \xi(s-d)+\mu] d s
\end{aligned}
$$

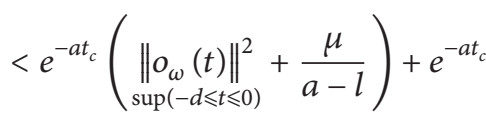





$$
\begin{aligned}
& \times \int_{0}^{t_{c}} e^{a s} \cdot e^{-\theta^{*}(s-d)} d s \\
& \left.+\frac{l \mu}{a-l} \int_{0}^{t_{c}} e^{a s} d s+\mu \int_{0}^{t_{c}} e^{a s} d s\right] .
\end{aligned}
$$

Combine the fact that $l e^{\theta^{*} d}=a-\theta^{*}$ and we can get

$$
\xi\left(t_{c}\right)<e^{-\theta^{*} t_{c}}\left\|o_{\omega}(t)\right\|^{2}+\frac{\mu}{a-l},
$$

which contradicts inequality (28). Thus, (27) can hold for $t>$ 0 .

$$
\begin{aligned}
& \text { As } \mu \rightarrow 0 \text {, then }
\end{aligned}
$$

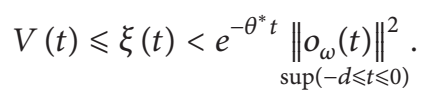


That is,

$$
\|\omega(t)\| \leqslant \underset{\sup (-d \leqslant t \leqslant 0)}{\left\|o_{\omega}(t)\right\|} e^{-\theta^{*} t / 2} .
$$

This completes the proof.

Remark 9. For the special case with only one leader, the final states of the followers will track the leader under the conditions in Theorem 8 . That is, $x_{i}(t) \rightarrow x_{l}(t)$, as $t \rightarrow \infty$, where $i \in \mathcal{N}$ and $x_{l}(t)$ is the state of the leader.

\subsection{Impulsive Containment Control for Nonlinear Dynamic} Systems of Switching Topology with Internal Delay. In the following, we propose the impulsive algorithms to achieve the containment control for nonlinear systems with switching topologies.

Consider the nonlinear system (1) in the switching cases; the proposed impulsive algorithm is

$$
\begin{array}{r}
u_{i}(t)=\sum_{k=1}^{\infty}\left[(-\beta) \sum_{j=1}^{M+N} a_{i j}(t)\left(x_{i}(t)-x_{j}(t)\right)\right] \delta\left(t-t_{k}\right), \\
i \in \mathcal{N},
\end{array}
$$

where $\beta>0$ is the feedback gain.

Using the impulsive algorithm (34), the system (1) can be rewritten as

$$
\begin{array}{r}
\dot{x}_{i}(t)=g\left(t, x_{i}, x_{i}(t-d)\right), \quad t \neq t_{k} \\
\Delta x_{i}(t)=(-\beta) \sum_{j=1}^{N+M} a_{i j}(t)\left(x_{i}(t)-x_{j}(t)\right), \\
t=t_{k}, i \in \mathcal{N}, \quad k=1,2, \ldots \\
\dot{x}_{i}(t)=g\left(t, x_{i}, x_{i}(t-d)\right), \quad t>0, i \in \mathscr{M},
\end{array}
$$

where $\Delta x_{i}\left(t_{k}\right)=x_{i}\left(t_{k}^{+}\right)-x_{i}\left(t_{k}\right)$ and assume that $x_{i}(t)$ is lefthand continuous at $t=t_{k}, k=1,2, \ldots, i \in \mathcal{N}$. Obviously, $x_{i}(t)$ is continuous when $t \in \mathscr{M}$. For simplicity, we assume that $x_{i}(t)$ is continuous at $t_{0}=0, i \in \mathscr{N}$.

Let

$$
\begin{aligned}
& x_{F}(t)= {\left[x_{M+1}(t), \ldots, x_{M+N}(t)\right]^{T}, } \\
& x_{L}(t)=\left[x_{1}(t), \ldots, x_{M}(t)\right]^{T}, \\
& G_{F}(t)=\left[g\left(t, x_{M+1}(t), x_{M+1}(t-d)\right), \ldots,\right. \\
&\left.g\left(t, x_{M+N}(t), x_{M+N}(t-d)\right)\right]^{T}, \\
& G_{L}(t)=\left[g\left(t, x_{M+1}(t), x_{M+1}(t-d)\right), \ldots,\right. \\
&\left.\quad g\left(t, x_{M+N}(t), x_{M+N}(t-d)\right)\right]^{T}, \\
& \omega(t)=x_{F}(t)+\left(L_{11}^{-1}(t) L_{12}(t)\right) x_{L}(t), \\
& G_{\omega}(t)=G_{F}(t)+\left(L_{11}^{-1}(t) L_{12}(t)\right) G_{L}(t) .
\end{aligned}
$$

From (4) and (35), we can arrive at

$$
\begin{aligned}
\dot{\omega}(t) & =G_{\omega}(t), \quad t \in\left(t_{k-1}, t_{k}\right] \\
\Delta \omega(t) & =-\beta L_{11}(t) \omega(t), \quad t=t_{k},
\end{aligned}
$$

where $k=1,2, \ldots$. The switching signal $\sigma: R^{+} \mapsto$ $\{1,2, \ldots, m\}$, which is equivalent to $\left(t_{k-1}, t_{k}\right] \mapsto L_{11}(t) \in$ $\left\{L_{11}^{(1)}, L_{11}^{(2)}, \ldots, L_{11}^{(m)}\right\}$, is a piecewise constant function. That is, the Lapacian matrix of the communication topologies is assumed to take from a set $\mathscr{L}=\left\{L^{(1)}, L^{(2)}, \ldots, L^{(m)}\right\}$ and the switching signal $\sigma: R^{+} \mapsto\{1,2, \ldots, m\}$ describes which communication topology is active in the time interval $\left(t_{k-1}, t_{k}\right]$. The communication topology is constant during $\left(t_{k-1}, t_{k}\right]$ and changes at $t_{k}, k=1,2, \ldots$ So, there exist $m$ subsystems in the system (37); that is,

$$
\begin{aligned}
& \dot{\omega}(t)=G_{\omega}(t), \quad t \in\left(t_{k-1}, t_{k}\right], \\
& \Delta \omega(t)=-\beta L_{11}^{\left(i_{k}\right)} \omega(t), \quad t=t_{k},
\end{aligned}
$$

where $i_{k} \in\{1,2, \ldots, m\}, k=1,2, \ldots$.

To simplify the presentation, define the constant numbers $\gamma$ that satisfy the following equality:

$$
\gamma=\max _{1 \leqslant i \leqslant m}\left\{\lambda_{\max }^{2}\left(I-\beta L_{11}^{(i)}\right)\right\}
$$

Theorem 10. Suppose that Assumption 2 is satisfied in every subsystem of (38) and Assumptions 1 and 6 are held. The system (1) with switching topologies using impulsive algorithm (34) achieves the containment control if

$$
\max _{1 \leqslant i \leqslant m}\left\{\lambda_{\max }^{2}\left(I-\beta L_{11}^{(i)}\right)\right\}<q^{2}
$$

That is,

$$
\begin{aligned}
& \frac{\max _{1 \leqslant i \leqslant m}\left\{\lambda_{\max }\left(L_{11}^{(i)}\right)\right\}-\min _{1 \leqslant i \leqslant m}\left\{\lambda_{\min }\left(L_{11}^{(i)}\right)\right\}}{\max _{1 \leqslant i \leqslant m}\left\{\lambda_{\max }\left(L_{11}^{(i)}\right)\right\}+\min _{1 \leqslant i \leqslant m}\left\{\lambda_{\min }\left(L_{11}^{(i)}\right)\right\}} \leqslant q<1, \\
& \frac{1-q}{\min _{1 \leqslant i \leqslant m}\left\{\lambda_{\min }\left(L_{11}^{(i)}\right)\right\}} \leqslant \beta \leqslant \frac{1+q}{\max _{1 \leqslant i \leqslant m}\left\{\lambda_{\max }\left(L_{11}^{(i)}\right)\right\}},
\end{aligned}
$$

$$
\tau_{0}<\frac{-\ln (q)}{2(K+l)},
$$

where $q$ is an auxiliary constant.

Proof. Construct a Lyapunov function $V(t)=\omega^{T}(t) \omega(t)$; then $V(t)$ is piece continuous and positive definite. It is obvious that $V(t)$ is left-hand continuous at $t=t_{k}, k=1,2, \ldots$. Now consider the time interval $\left(t_{k-1}, t_{k}\right], k=1,2, \ldots$. When $t \in$ $\left(t_{k-1}, t_{k}\right]$, owing to Assumption 1, the differential of the $V(t)$ with regard to (38) is

$$
\begin{aligned}
\dot{V}(t) & =2 \omega^{T}(t) \dot{\omega}(t)=2 \bar{\omega}^{T}(t) \bar{G}_{\omega}(t) \\
& \leqslant 2 \bar{\omega}^{T}(t)(K \bar{\omega}(t)+l \bar{\omega}(t-d)),
\end{aligned}
$$


where $\bar{G}_{\omega}(t)=\left[\left|\dot{\omega}_{1}(t)\right|, \ldots,\left|\dot{\omega}_{N}(t)\right|\right]^{T}$. It is obvious that

$$
2 \bar{\omega}^{T}(t) \bar{\omega}(t-d) \leqslant \bar{\omega}^{T}(t) \bar{\omega}(t)+\bar{\omega}^{T}(t-d) \bar{\omega}(t-d) .
$$

As a consequence, we obtain

$$
\dot{V}(t) \leqslant(2 K+l) V(t)+l V(t-d), \quad t \in\left(t_{k-1}, t_{k}\right] .
$$

It follows from (38) that $\omega\left(t_{k}^{+}\right)=\left(I-\beta L_{11}^{\left(i_{k}\right)}\right) \omega\left(t_{k}\right)$. Combine the definition of $V(t)$ and $\gamma$ in equality (39); we can obtain

$$
\begin{aligned}
V\left(t_{k}^{+}\right) & =\omega^{T}\left(t_{k}^{+}\right)\left(I-\beta L_{11}^{\left(i_{k}\right)}\right)^{2} \omega\left(t_{k}^{+}\right) \\
& \leqslant \lambda_{\max }^{2}\left(I-\beta L_{11}^{\left(i_{k}\right)}\right) \omega^{T}\left(t_{k}\right) \omega\left(t_{k}\right) \leqslant \gamma V\left(t_{k}\right),
\end{aligned}
$$

where $k=1,2, \ldots, i_{k} \in\{1,2, \ldots, m\}$.

To prove the Lyapunov function $V(t)$ is convergent, we construct an auxiliary variable $\xi$ in the following form:

$$
\begin{aligned}
\dot{\xi}(t) & =(2 K+l) \xi(t)+l \xi(t-d)+\mu, \quad t \neq t_{k}, \\
\xi\left(t_{k}^{+}\right) & =\lambda_{\max }^{2}\left(I-\beta L_{11}^{\left(i_{k}\right)}\right) \xi\left(t_{k}\right),
\end{aligned}
$$

where the initial states of $\xi(t)=\left\|o_{\omega}(t)\right\|^{2},-d \leqslant t \leqslant 0, \mu>0$, $k=1,2, \ldots$, where $o_{\omega}(t)=o_{F}(t)+\left(L_{11}^{-1}(0) L_{12}(0)\right) o_{L}(t), o_{F}(t)=$ $\left[o_{M+1}(t), \ldots, o_{M+N}(t)\right]^{T}, o_{L}(t)=\left[o_{1}(t), \ldots, o_{M}(t)\right]^{T}$.

Let

$$
\begin{gathered}
\dot{\eta}(t)=(2 K+l) \eta(t), \quad t \neq t_{k} \\
\eta\left(t_{k}^{+}\right)=\lambda_{\max }^{2}\left(I-\beta L_{11}^{\left(i_{k}\right)}\right) \eta\left(t_{k}\right),
\end{gathered}
$$

where $k=1,2, \ldots$

From (45), (46), and (47), it is obvious that, for all $t>0$, $0 \leqslant V(t) \leqslant \xi(t)$. And the solution of (47) satisfies the following integral equation:

$$
\xi(t)=\phi(t, 0) \xi(0)+\int_{0}^{t} \phi(t, s)[l \xi(s-d)+\mu] d s,
$$

where $\phi(t, s),(t, s \geqslant 0)$, is the state transition matrix of the system (48).

When $t_{k}<t<t_{k+1}$ and $t_{k-1}<s<t_{k}$, we can arrive at

$$
\phi(t, s) \leqslant e^{(2 K+l)(t-s)} \lambda_{\max }^{2}\left(I-\beta L_{11}^{\left(i_{k}\right)}\right) .
$$

From inequalities (40) and (41), we can obtain

$$
\left|\lambda_{\max }\left(I-\beta L_{11}^{\left(i_{k}\right)}\right)\right| \leqslant q<1 .
$$

Let $a=-(2 K+l)-\gamma / 2$ and $2 \ln (q) / \tau_{0}=\gamma$. Then, from inequality (42), we can get $\gamma<-4(K+l)$ and $a>0$. Accordingly, inequality (50) can be rewritten as follows:

$$
\begin{aligned}
\phi(t, s) & \leqslant e^{(-a-\gamma / 2)(t-s)} \lambda_{\max }^{2}\left(I-\beta L_{11}^{\left(i_{k}\right)}\right) \\
& <e^{-a(t-s)} \cdot e^{-(\gamma / 2) \cdot 2 \tau_{0}} \lambda_{\max }^{2}\left(I-\beta L_{11}^{\left(i_{k}\right)}\right)=e^{-a(t-s)} .
\end{aligned}
$$

Thus, from (49), we can obtain

$$
\xi(t)<e^{-a t} \| \underset{\sup (-d \leqslant t \leqslant 0)}{\left\|o_{\omega}(t)\right\|^{2}}+\int_{0}^{t} e^{-a(t-s)}[l \xi(s-d)+\mu] d s .
$$

Let $\varepsilon(\theta)=\theta-a+l e^{\theta d}$. Then, $\varepsilon(\theta)=0$ has a unique solution $\theta^{*}>0$ because $\varepsilon(\theta)$ is a strictly monotonic function $\dot{\varepsilon}(\theta)=$ $1+l d e^{\theta d}>0$ and $\varepsilon(0)=-a+l<0, \varepsilon(+\infty)>0$. It is obvious that the following inequality is satisfied:

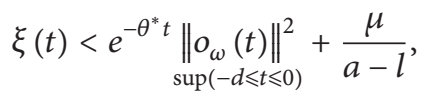

where $-d \leqslant t \leqslant 0$. Then we can prove that inequality

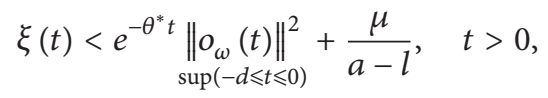

is true in the following.

Suppose that inequality (55) is not held; there must be a $t_{c}$ such that

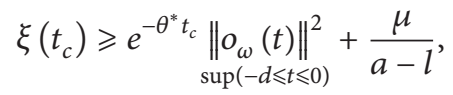



However, from (53) and (57), we can obtain

$$
\xi\left(t_{c}\right)<e^{-\theta^{*} t_{c}} \underset{\substack{\sup (-d \leqslant t \leqslant 0) \\ \sup }}{\|(t)\|^{2}}+\frac{\mu}{a-l}
$$

which contradict inequality (56). Thus, (55) can hold for $t>$ 0.

As $\mu \rightarrow 0$,

$$
V(t) \leqslant \xi(t)<e^{-\theta^{*} t} \underset{\substack{\sup (-d \leqslant t \leqslant 0) \\ o_{\omega}(t) \|^{2}}}{ }
$$

That is,

$$
\|\omega(t)\| \leqslant \underset{\sup (-d \leqslant t \leqslant 0)}{\left\|o_{\omega}(t)\right\|} e^{-\theta^{*} t / 2}
$$

This completes the proof.

Remark11. For the special case with only one leader, the result obtained in Theorem 10 also holds. And the final states of the followers will track the leader's state.

Remark 12. The fixed communication topology in Section 2.1 can be viewed as a special case in the switching topology case. The achievement of the containment control in the fixed topology systems is the fundamental problem and it contributes to the proof procedure of the switching counterpart.

Remark 13. The design procedure of the impulsive controller in Theorem 8 is that first, according to the communication topology of the graph $\mathscr{G}$ and inequality (11), the region of $q$ can be ascertained. Then, we choose an appropriate constant $q$. From conditions (12) and (13), we can derive the region of the feedback $\alpha$ and the impulsive period $\tau_{0}$. Choosing the appropriate $\alpha$ and $\tau_{0}$, the containment control can be achieved. The counterpart under switching topology in Theorem 10 is similar and we omit it. 




(1)



(3)

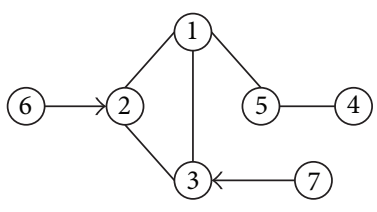

(2)

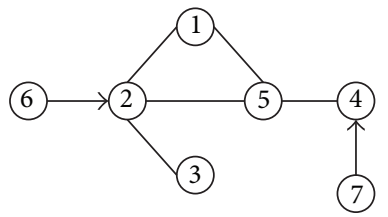

(4)
FIGURE 1: The interaction graphs.

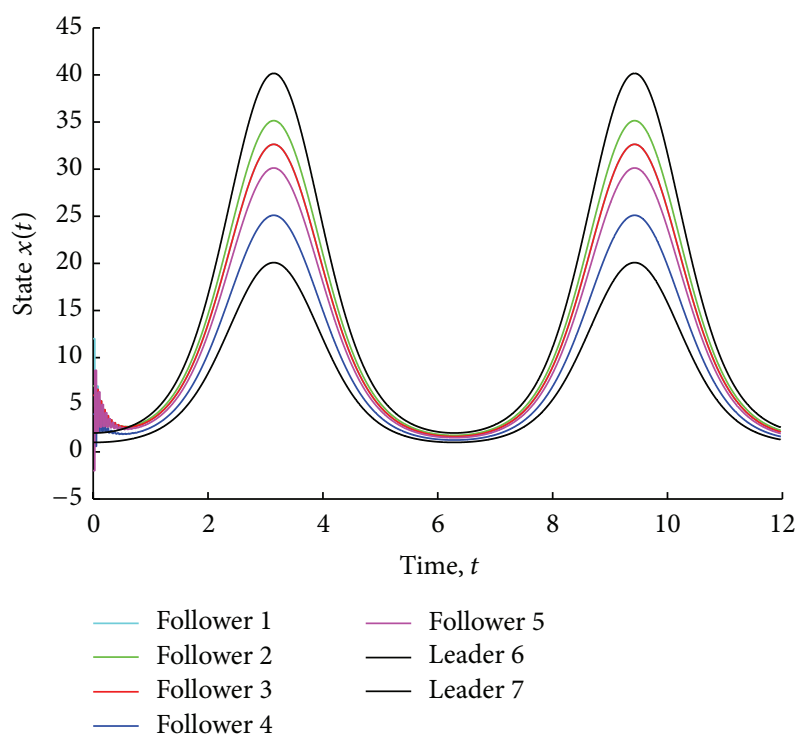

FIGURE 2: The states of the agents in system (1) with fixed topology using impulsive algorithm (6).

\section{Simulations}

In this section, we give some examples to verify the theoretical analysis. The communication graphs are shown in Figure 1. The agents labeled as 6 and 7 are the leaders and the agents labeled as 1, 2, 3, 4, and 5 are the followers.

Example 1. According to Figure 1, the communication graph (1) satisfies the Assumption 2. Consider that the system (1) with the nonlinear dynamic is $g\left(t, x_{i}(t), x_{i}(t-d)\right)=$ $x_{i}(t) \sin (t)+0.5 x_{i}(t) \sin (t-d), i \in \mathscr{V}$. Obviously, Assumption 1 is satisfied in the system and the corresponding parameters are $K=1$ and $l=0.5$, respectively. Using the proposed impulsive containment control algorithm (6), the simulation results are shown in Figure 2. From condition (11) in Theorem 8 , the regions of $q$ should be $0.8774<q<1$. In this case, we choose $q=0.9$ and, according to conditions (12) and (13), it is obvious that the region of $\alpha$ and the impulsive period $\tau_{0}$ are $0.3263 \leqslant \alpha \leqslant 0.4048$ and $\tau_{0}<0.0351$,

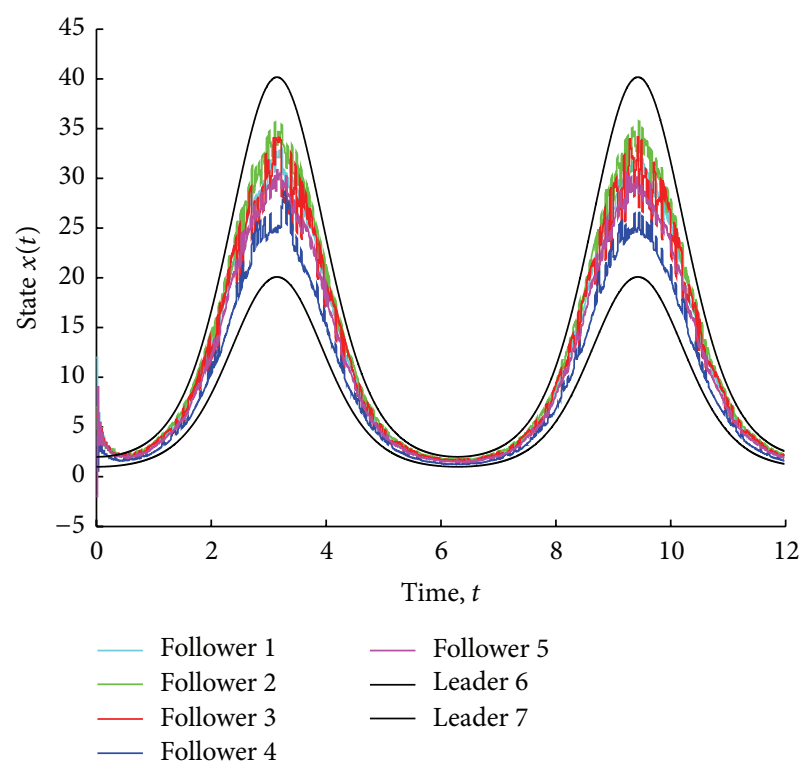

FIGURE 3: The states of the agents in system (1) with switching topologies using impulsive algorithm (34).

respectively. We choose $\alpha=0.38$ and $\tau_{0}=0.03$. Suppose the time-delay $d=0.01$. Obviously, Assumption 6 is satisfied in the system (1). From Figure 2, it is obvious that the final states of the followers converge into the convex hull formed by the leaders.

Example 2. The graphs of the communication topology are shown in Figure 1. Now consider the nonlinear system $g\left(t, x_{i}(t), x_{i}(t-d)\right)=x_{i}(t) \sin (t)+0.5 x_{i}(t) \sin (t-d), i \in \mathscr{V}$, with switching topologies. It is clear that the four topologies in Figure 1 satisfy Assumption 2 and the dynamics of the system satisfy Assumption 1 . In every time interval $\left(t_{k-1}, t_{k}\right]$, $k=1,2, \ldots$, the communication topology is chosen from Figure 1 randomly. Using the impulsive algorithm (34), the simulation result of the nonlinear system with switching topologies is shown in Figure 3. According to the conditions in Theorem 10, the region of $q$ is $q>0.9273$ and we choose $q=0.93$. According to condition (41), the feedback gain $\beta$ should choose from $0.3755 \leqslant \beta \leqslant 0.3907$ and we choose $\beta=$ 0.38 . From inequality (42), the impulsive period $\tau_{0}$ should be less than 0.0242 and we choose $\tau_{0}=0.02$. And suppose that the time-delay $d=0.01$, which satisfies Assumption 6 . From Figure 3, we can get that the containment control is achieved and the follower's states converge into the convex hull spanned by the multiple leaders. The final states of the followers $x_{F}(t)$ converge to $\left(-L_{11}^{-1}(t) L_{12}(t)\right) x_{L}(t)$ as $t \rightarrow$ $+\infty$ and the states $\left(-L_{11}^{-1}(t) L_{12}(t)\right) x_{L}(t)$ are time varying and nonsmooth.

\section{Conclusions}

This paper has considered the nonlinear multiagent systems with time-delay of multiple leaders. When the topologies in the nonlinear systems are fixed, an impulsive control 
algorithm is proposed and some useful sufficient conditions to achieve containment control are obtained. Also, the nonlinear systems under switching topologies are considered. Using the proposed impulsive algorithms, the final states of all the followers will converge into the convex hull formed by the states of the leaders. Finally, some simulations are given to illustrate our theoretical analyses.

\section{Conflict of Interests}

The authors declare that there is no conflict of interests regarding the publication of this paper.

\section{Acknowledgments}

The work was supported partly by the National Natural Science Foundation of China under Grants 61374064, 61304152, and 61364017, the National Key Technology R\&D Program of China 2013BAA01B01, and the China Postdoctoral Science Foundation funded Project 2012M511258 and 2013T60738.

\section{References}

[1] H. Su, X. Wang, and Z. Lin, "Flocking of multi-agents with a virtual leader," IEEE Transactions on Automatic Control, vol. 54, no. 2, pp. 293-307, 2009.

[2] H. Du and S. Li, "Attitude synchronization control for a group of flexible spacecraft," Automatica, vol. 50, no. 2, pp. 646-651, 2014.

[3] D. Yuan, D. W. Ho, and S. Xu, "Inexact dual averaging method for distributed multi-agent optimization," Systems \& Control Letters, 2014.

[4] Z. Liu, Z. Guan, T. Li, X. Zhang, and J. Xiao, "Quantized consensus of multi-agent systems via broadcast gossip algorithms," Asian Journal of Control, vol. 14, no. 6, pp. 1634-1642, 2012.

[5] X. Lu, F. Austin, and S. Chen, "Formation control for secondorder multi-agent systems with time-varying delays under directed topology," Communications in Nonlinear Science and Numerical Simulation, vol. 17, no. 3, pp. 1382-1391, 2012.

[6] R. Olfati-Saber and R. M. Murray, "Consensus problems in networks of agents with switching topology and time-delays," IEEE Transactions on Automatic Control, vol. 49, no. 9, pp. 15201533, 2004.

[7] W. Ren and R. W. Beard, "Consensus seeking in multiagent systems under dynamically changing interaction topologies," IEEE Transactions on Automatic Control, vol. 50, no. 5, pp. 655661,2005

[8] W. Zhu and D. Cheng, "Leader-following consensus of secondorder agents with multiple time-varying delays," Automatica, vol. 46, no. 12, pp. 1994-1999, 2010.

[9] H. Su, M. Z. Q. Chen, J. Lam, and Z. Lin, "Semi-global leaderfollowing consensus of linear multi-agent systems with input saturation via low gain feedback," IEEE Transactions on Circuits and Systems I: Regular Papers, vol. 60, no. 7, pp. 1881-1889, 2013.

[10] H. Su, M. Chen, X. Wang, and J. Lam, "Semiglobal observerbased leader-following consensus with input saturation," IEEE Transactions on Industrial Electronics, vol. 61, pp. 2842-2850, 2014.

[11] X. Lu, R. Lu, S. Chen, and J. Lu, "Finite-time distributed tracking control for multi-agent systems with a virtual leader," IEEE
Transactions on Circuits and Systems I: Regular Papers, vol. 60, no. 2, pp. 352-362, 2013.

[12] Y. Lou and Y. Hong, "Target containment control of multi-agent systems with random switching interconnection topologies," Automatica, vol. 48, no. 5, pp. 879-885, 2012.

[13] Z. Li, Z. Duan, W. Ren, and G. Feng, "Containment control of linear multi-agent systems with multiple leaders of bounded inputs using distributed continuous controllers," International Journal of Robust and Nonlinear Control, 2014.

[14] H. Liu, G. Xie, and L. Wang, "Containment of linear multiagent systems under general interaction topologies," Systems and Control Letters, vol. 61, no. 4, pp. 528-534, 2012.

[15] J. Li, W. Ren, and S. Xu, "Distributed containment control with multiple dynamic leaders for double-integrator dynamics using only position measurements," IEEE Transactions on Automatic Control, vol. 57, no. 6, pp. 1553-1559, 2012.

[16] Z. Meng, W. Ren, and Z. You, "Distributed finite-time attitude containment control for multiple rigid bodies," Automatica, vol. 46, no. 12, pp. 2092-2099, 2010.

[17] H. Liu, G. Xie, and L. Wang, "Necessary and sufficient conditions for containment control of networked multi-agent systems," Automatica, vol. 48, no. 7, pp. 1415-1422, 2012.

[18] J. Mei, W. Ren, and G. Ma, "Distributed containment control for Lagrangian networks with parametric uncertainties under a directed graph," Automatica, vol. 48, no. 4, pp. 653-659, 2012.

[19] Y. Cao, W. Ren, and M. Egerstedt, "Distributed containment control with multiple stationary or dynamic leaders in fixed and switching directed networks," Automatica, vol. 48, no. 8, pp. 1586-1597, 2012.

[20] P. Wang and Y. Jia, "Distributed containment control of secondorder multi-agent systems with inherent non-linear dynamics," IET Control Theory \& Applications, vol. 8, no. 4, pp. 277-287, 2014.

[21] K. Liu, G. Xie, W. Ren, and L. Wang, "Consensus for multiagent systems with inherent nonlinear dynamics under directed topologies," Systems and Control Letters, vol. 62, no. 2, pp. 152162, 2013.

[22] W. Yu, G. Chen, and M. Cao, "Consensus in directed networks of agents with nonlinear dynamics," IEEE Transactions on Automatic Control, vol. 56, no. 6, pp. 1436-1441, 2011.

[23] Z. Liu, H. Zhou, Z. Guan, W. Hu, L. Ding, and W. Wang, "Distributed impulsive consensus of the multiagent system without velocity measurement," Abstract and Applied Analysis, vol. 2013, Article ID 825307, 8 pages, 2013.

[24] J. Lu, J. Kurths, J. Cao, N. Mahdavi, and C. Huang, "Synchronization control for nonlinear stochastic dynamical networks: pinning impulsive strategy," IEEE Transactions on Neural Networks and Learning Systems, vol. 23, no. 2, pp. 285-292, 2012.

[25] Z. Guan, D. J. Hill, and X. Shen, "On hybrid impulsive and switching systems and application to nonlinear control," IEEE Transactions on Automatic Control, vol. 50, no. 7, pp. 1058-1062, 2005.

[26] Z.-W. Liu, Z.-H. Guan, X. Shen, and G. Feng, "Consensus of multi-agent networks with aperiodic sampled communication via impulsive algorithms using position-only measurements," Institute of Electrical and Electronics Engineers, vol. 57, no. 10, pp. 2639-2643, 2012.

[27] Z.-H. Guan, Z.-W. Liu, G. Feng, and Y.-W. Wang, "Synchronization of complex dynamical networks with time-varying delays via impulsive distributed control," IEEE Transactions on Circuits and Systems I: Regular Papers, vol. 57, no. 8, pp. 2182-2195, 2010. 
[28] G. Wen, Z. Duan, W. Yu, and G. Chen, "Consensus of secondorder multi-agent systems with delayed nonlinear dynamics and intermittent communications," International Journal of Control, vol. 86, no. 2, pp. 322-331, 2013.

[29] R. T. Rockafellar, Convex Analysis, vol. 28 of Princeton Mathematical Series, Princeton University Press, Princeton, NJ, USA, 1997. 


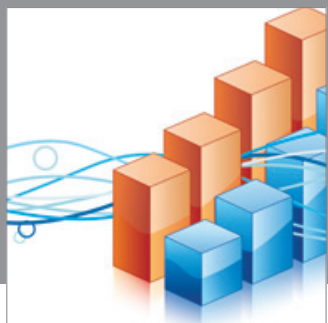

Advances in

Operations Research

mansans

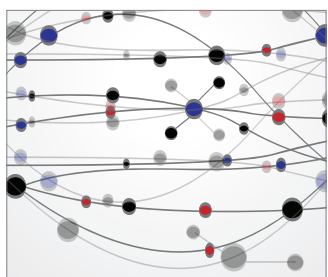

The Scientific World Journal
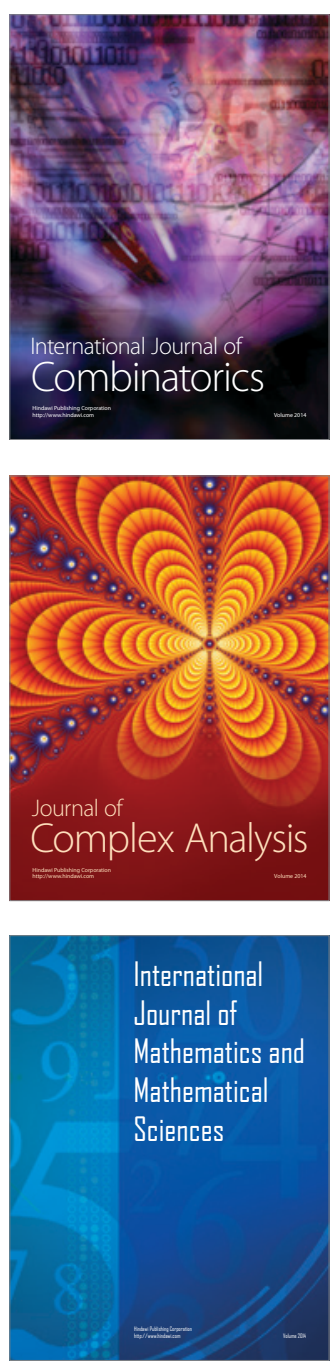
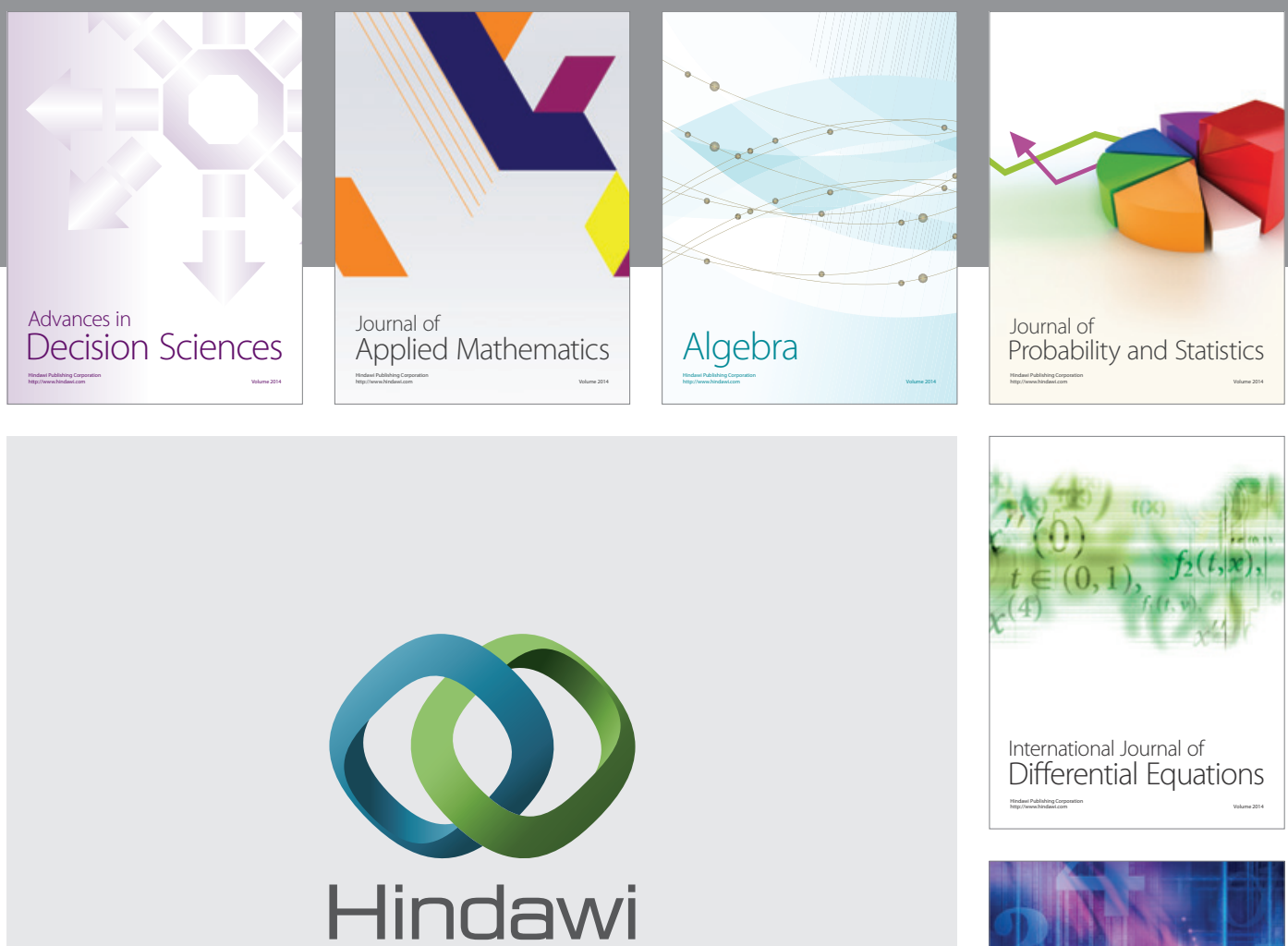

Submit your manuscripts at http://www.hindawi.com
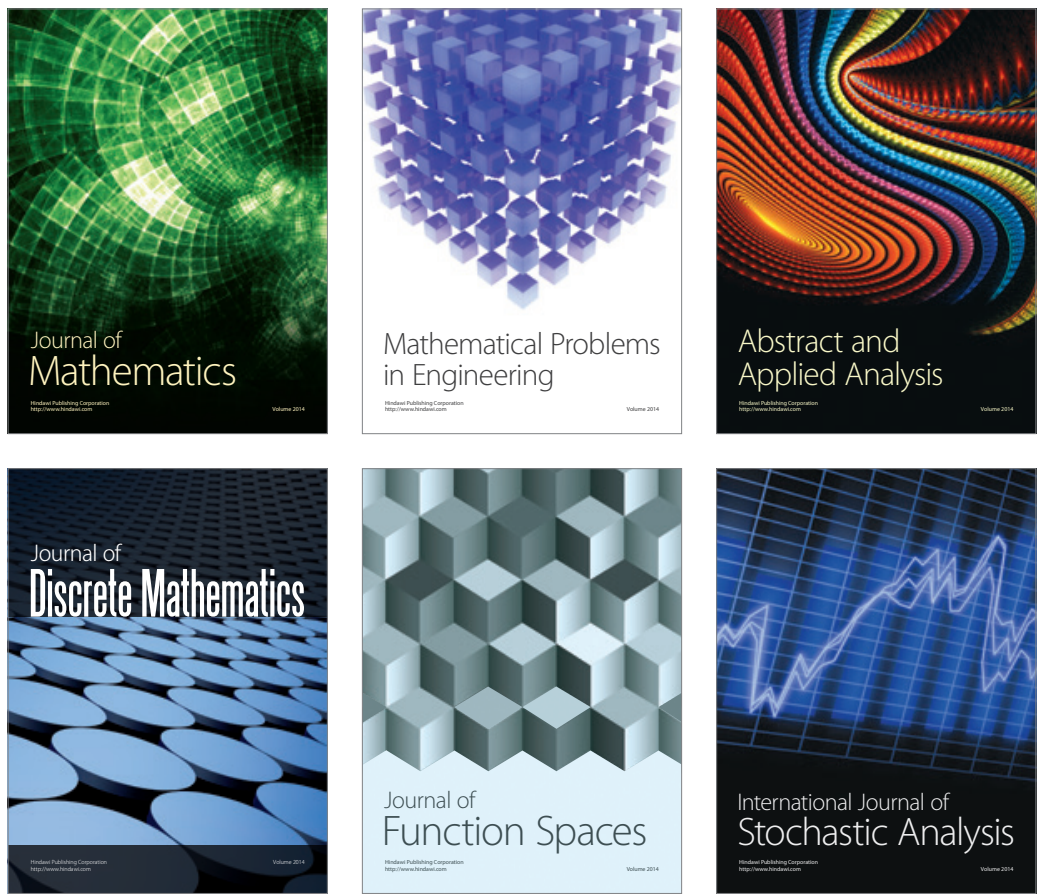

Journal of

Function Spaces

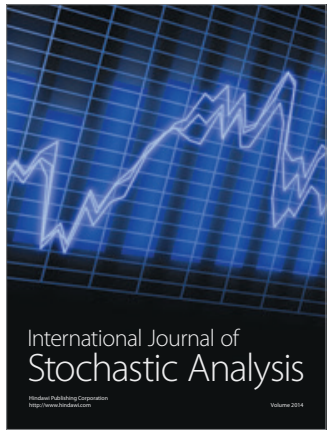

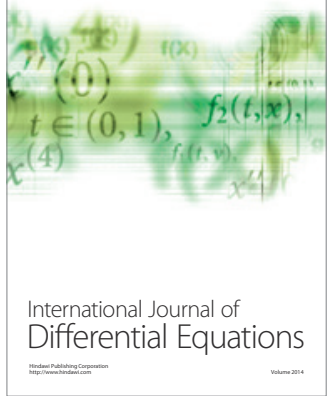
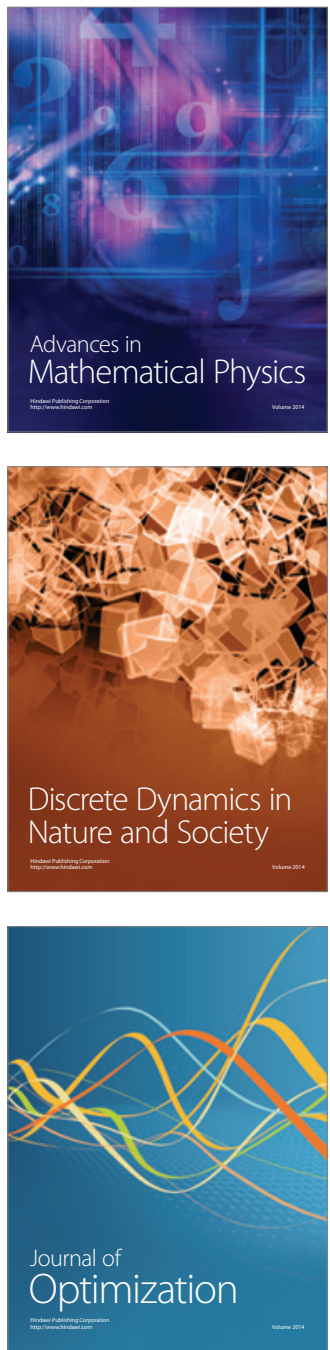